\title{
Evaluation of Primary and Secondary Free Flap Desyndactylization Techniques in Hand and Digit Reconstruction: A Systematic Review
}

\author{
Michael B. Gehring, MD ${ }^{1}$ Matthew L. Iorio, MD ${ }^{1}$ \\ ${ }^{1}$ Division of Plastic and Reconstructive Surgery, University of \\ Colorado Anschutz Medical Center, Aurora, Colorado \\ J Reconstr Microsurg Open 2020;5:e107-e114.
}

\begin{abstract}
Address for correspondence Matthew L. Iorio, MD, Division of Plastic and Reconstructive Surgery, University of Colorado, Anschutz Medical Center, 12631 East 17th Avenue, Aurora, CO 80045

(e-mail: matt.iorio@cuanschutz.edu).
\end{abstract}

\begin{abstract}
Keywords

- surgical syndactylization

- multilobe free flaps

- hand reconstruction

Objective Injuries of the hand often require free flap reconstruction. To minimize flap loss, evidence exists to surgically syndactylize digits when repairing multiple injuries, with delayed flap division, or desyndactylization. However, evidence suggests that division of the flap at the time of inset can be accomplished with minimal negative effect. The purpose of this study was to evaluate outcomes, following hand reconstruction with free flaps utilizing either acute or staged desyndactylization techniques. Methods A systematic review utilizing the Preferred Reporting Items for Systematic Reviews and Meta-Analyses (PRISMA) guidelines was performed. Articles that described the use of a free flap for surgically syndactylized digits or multilobed flaps for coverage of multiple digits were included.

Results One hundred sixty-one articles were reviewed with 34 fulfilling inclusion criteria. One hundred seventeen patients underwent 145 free flap reconstructions. Traumatic avulsions (49\%) were the most common injuries, followed by burns (11\%). Twenty-one (62\%) papers described surgical syndactylization of digits, which were later desyndactylized and five (15\%) papers included reconstruction of more than one digit with multilobed free flaps. Eight papers (24\%) described both techniques. Overall, $100 \%$ of included flaps survived. Total complication rate was $6 \%$, with six complications $(67 \%)$ occurring in flaps with primary syndactylization.

Conclusion Hand defects often require free flaps for reconstruction. Although free flaps for the reconstruction of digital defects is technically demanding, they result in better outcomes. With available evidence indicating complications rates less than those of staged desyndactylization, multidigit reconstruction with multilobed free flaps may be a more desirable technique.
\end{abstract}

Traumatic injuries, infections, and oncologic resections of the hand and fingers often require reconstruction of more than one digit or web space for adequate function. In the setting of a major injury or burn, the syndactylization can occur through direct scarring and fibrosis resulting in a limited range of finger motion with poor function.

Immediate coverage of hand soft tissue defects with free flaps has been demonstrated as a safe and viable option for the past three decades. ${ }^{1-3}$ Indications for free flap hand coverage can include clinical scenarios in which skin grafts or locoregional flaps would be unsuitable, either from a durability standpoint, or in those instances where scar tissue would either preclude future exploration of the site (e.g., tendon grafting) and reduce active motion and function. ${ }^{4}$ Reconstructive free flap coverage of the hand has several benefits including coverage with composite reconstruction of damaged or received

September 22, 2020

accepted

October 27, 2020
DOI https://doi.org/

10.1055/s-0040-1721705. ISSN 2377-0813.
Copyright $\odot 2020$ by Thieme Medical Publishers, Inc., 333 Seventh Avenue, 18th Floor, New York, NY 10001, USA Tel: +1(212) 760-0888.
License terms

(요 (1) $\Theta \circledast$ 
absent tissues and early mobilization to aid in restoration of function. ${ }^{1}$ Free flaps can be harvested in almost any size and carry their own blood supply with angiogenic and lymphogenic potential to improve venous and lymphatic drainage of the traumatized region., ${ }^{5,6}$ In the setting of multiple finger or webspace injuries, there are several described techniques utilizing free flaps for reconstruction.

One technique consists of reconstruction that initially utilizes syndactylization to allow the flap and soft tissues to heal, with secondary "desyndactylization" or separation of the fingers and contouring of the webspace. Syndactylization of digits for the reconstruction is often utilized with free flaps to preserve not only the affected digits, but also the length of injured digits by providing well-vascularized coverage to devitalized tissue. ${ }^{7}$ Syndactylization of multifinger soft tissue defects have been reported to have disadvantages of requiring secondary division operations with high risk of flap necrosis. ${ }^{8,9}$ However, several studies have demonstrated good functional hand outcomes with both arterialized venous and arterial free flaps after multifinger syndactylization and subsequent desyndactylization. ${ }^{10-14}$

In comparison, another reconstructive technique utilizes primary desyndactylization of the fingers and web spaces by dividing the flap at the time of coverage. Perforator flaps or multilobed flaps are the workhouse flaps for this technique. Anatomically, any musculocutaneous perforator flap that is perfused by more than one perforator can be split into multiple cutaneous flaps, based on the number of the perforators. ${ }^{15}$ Each skin paddle is nourished by at least one single musculocutaneous or septocutaneous perforator, with all the perforators deriving from the same trunk vessel. ${ }^{15}$ By having a single, common trunk vessel, the hand surgeon can make a single arterial and venous microanastomosis while simultaneously providing coverage to two- or more-digit defects, which otherwise may have required separate microanastomoses for each digit defect. ${ }^{8,16-18}$

However, with the potential for flap loss, tissue necrosis, or web space creep, the optimal techniques for free flap reconstruction of more than one-digit defect have not been identified. The aim of this study was to determine the safety and patient outcomes of either technique for simultaneous reconstruction of more than one-digit defect or web space with free tissue transfers, as well as a review of the current literature and available techniques.

\section{Methods}

A systematic review utilizing the Preferred Reporting Items for Systematic Reviews and Meta-Analyses (PRISMA) guidelines ${ }^{19}$ was performed, utilizing studies from PubMed, Embase, and Google Scholar. Dates of search inclusion were from 1992 to 2020. Articles that described the use of a free flap for hand reconstruction that surgically syndactylized digits and were later desyndactylized, or described multilobed free flaps for the coverage of multiple digits were included. For the purposes of this paper, the authors defined "desyndactylization" as a secondary procedure in which at least two previously conjoined digits were separated by incising the free flap construct. ${ }^{11}$
Additional inclusion criteria included (1) upper extremity defect; (2) availability of clinical data of outcomes or complications; and (3) soft tissue reconstruction by free flap transfer as the reconstructive method. Exclusion criteria included: (1) overlapping articles; (2) free flap reconstruction techniques that did not involve two or more digits; (3) use of free fascial or omental flaps; and (4) unavailable clinical data of outcomes or complications. Only articles published in English were included.

\section{Results}

The search yielded 162 studies for abstract review. The PRISMA flow chart is demonstrated in - Fig. 1. Of these abstracts, two studies were excluded for not being published in English, and an additional five articles were not included as the articles did not include descriptions of surgical techniques or outcomes. Thirty-four articles met final inclusion criteria. There were no systematic reviews or Cochrane reviews. No randomized control trials were found. - Supplementary Table S1 summarizes the included articles, flap type, complications, and other key findings reported by the articles' authors.

A total of 117 patients underwent 145 free flap reconstructions. Traumatic avulsions and degloving injuries $(n=71,49 \%)$ were the most common injuries, followed by burns $(n=16$, 11\%). Twenty-one articles ${ }^{7,9-13,20-34}$ described surgical syndactylization of digits, which were later desyndactylized and five articles $^{35-39}$ included reconstruction of more than one digit with multilobed free flaps. Eight articles $8,15,40-45$ described both techniques.

Arterialized venous free flaps were only described in the surgical syndactylization cohort and accounted for 11 of all the free flaps included in the study. Nine of these flaps were harvested from the volar forearm with the remaining two flaps harvested from the dorsal foot. Although arterialized venous free flaps compromised only $8 \%$ of the flaps in this systematic review, they accounted for $50 \%$ of the complications. Eleven articles described dorsal web space reconstructions $(n=103)$ in 58 patients. Overall, $85 \%$ of the web spaces were reconstructed with desyndactylized flaps. Total 6 articles described tendon reconstruction techniques and 11 articles involved sensate flap reconstruction requiring nerve coaptation.

\section{Surgical Syndactylization with Free Flap Cohort}

There were 46 free flaps in the surgical syndactylization cohort with volar forearm, arterialized venous flaps $(n=9)$ most frequently used, followed by anterolateral thigh (ALT) flaps $(n=8)$. Least frequently used flaps included use of a spare part, osteofasciocutaneous chimeric fibular flap $(n=1)$, and another article reporting use of a fasciocutaneous, prefabricated serratus fascia flap $(n=1)$. There were five reports of sensate free flaps and one case of digital nerve repair. Desyndactylization occurred at an average of 10.1 months (range $=3-24$ weeks). Studies that did not have any complications averaged desyndacytlization at 10.5 months, while studies reporting a flap complication averaged desyndactylization at 10.6 months. Three studies did not include desyndactylization time.

The average follow-up in this cohort was 14 months. Most articles reported adequate closure of the hand defect with 


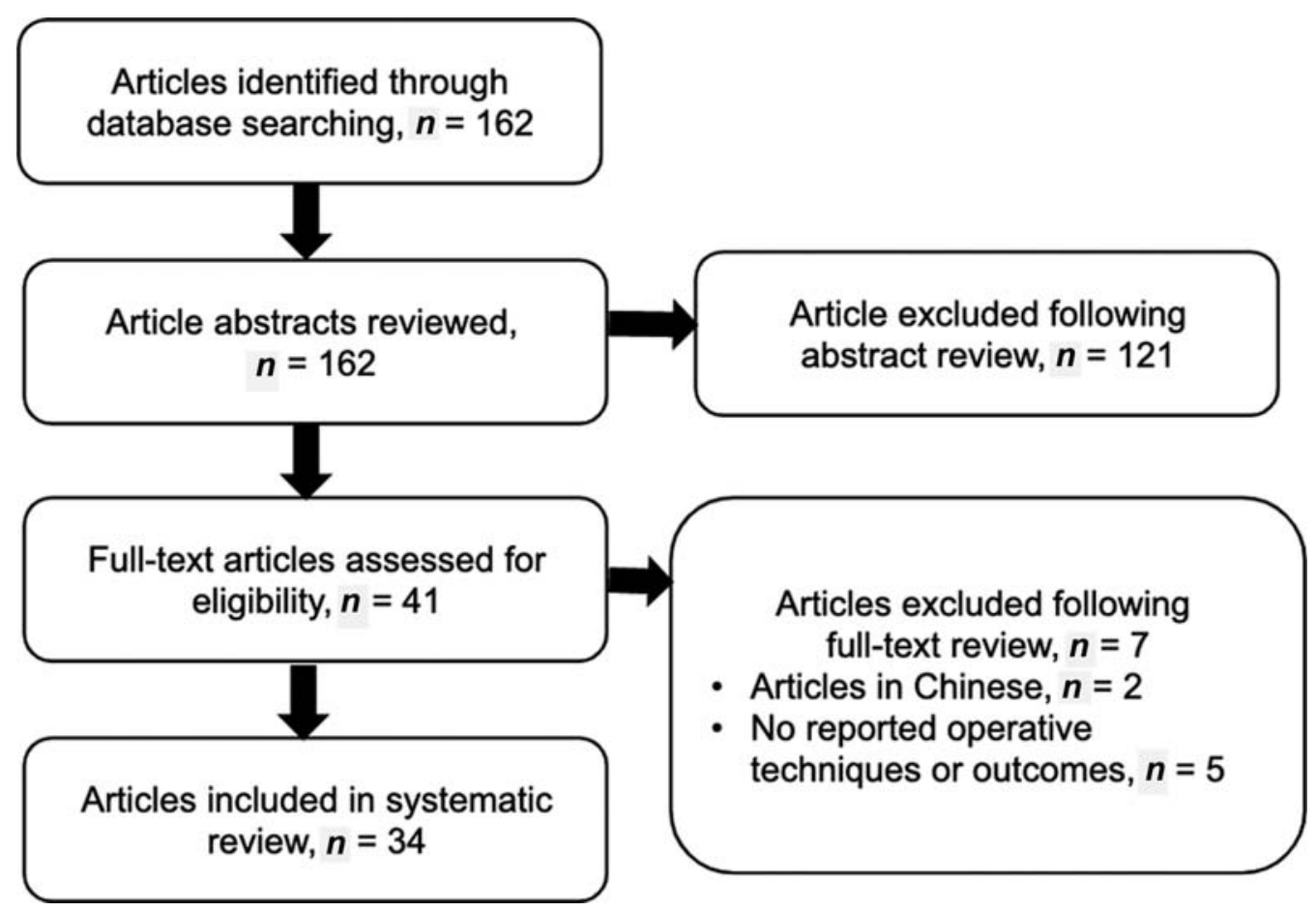

Fig. 1 Preferred Reporting Items for Systematic Reviews and Meta-Analyses flow diagram.

improved hand functionality and aesthetics. Only one study reported objective outcomes using the QuickDASH score, which averaged 18.2 (range $=5.8-52.5)$. Total active range of motion averaged 173.35 degrees in the two studies that reported it and $61.5 \%$ in another study. Grip strength in the two reporting studies averaged 69\% when compared with the unaffected hand. Two-point discrimination was reported in 10 articles with an average of $12.6 \mathrm{~mm}$ for static and an average of $9.5 \mathrm{~mm}$ for moving. Five articles reported the restoration of protective sensation in the reconstructed hand defects.

Although there was $100 \%$ flap survival rate in this cohort, there were six flap complications. Complications included epidermolysis, partial flap necrosis, scar contracture with partial bone resorption, and sloughed tissue resulting in exposed hardware. All complications were managed conservatively except for the case of sloughed tissue with exposed hardware in the combined lateral arm flap with ALT flap case report, which was treated with a split-thickness skin graft and free medial sural artery perforator flap. Volar forearm arterialized venous free flaps accounted for the highest amount of flap complications $(n=3)$. There were two donor site complications which included partial skin graft loss and a widened scar.

\section{Multilobed Free Flap Cohort}

The multilobed free flap cohort most frequently used the multilobed ALT flap $(n=48)$, followed by the multilobed chimeric dorsalis pedis flap $(n=15)$. The least frequently used flap construct was a conjoint flap consisting of the dorsalis pedis flap with second toe flap fillet and trimmed toe flap $(n=1)$. Thirty-four sensate free flaps were used in this cohort, with $97 \%$ of them being innervated ALT flaps. One article described use of sensate combined medialis pedis and medial plantar free flaps based off the medial plantar nerve branches in three patients.

This cohort averaged 9.3-month follow-up with all articles reporting satisfactory appearance and function of the reconstructed hand defects. No studies reported objective outcomes using validated hand surveys. Average degrees of movement for MPJ flexion, wrist flexion and dorsiflexion, and thumb abduction were reported in one study which significantly improved after reconstruction. One study reported an average static two-point discrimination of $6.5 \mathrm{~mm}$ with another study reporting restoration of protective sensation in all reconstructed digits.

There was $100 \%$ flap survival in this cohort. Only one study reported a complication of index and middle finger proximal interphalangeal joint contracture (30-degree flexion). No donor site complications were reported.

\section{Cohort Describing Both Techniques}

Of the articles describing both surgical syndactylization and multilobed free flap reconstruction techniques, hand injuries tended to be more severe and involved most digits necessitating larger and more free flaps for coverage. Superficial circumflex iliac artery flaps were most frequently used $(n=8)$, followed by multilobed ALT flaps $(n=7)$ and scapular lobulated combined flaps $(n=7)$. The combined thoracodorsal flap with serratus fascia was least frequently used $(n=1)$.

Average follow-up was 9.6 months. QuickDASH was reported in one study, averaging 26.8 (range 16-34) and the Michigan Hand Outcomes survey was reported in another study with one patient scoring 5 out of 5 , two patients scoring 4 out of 5 , and five patients scoring 2 out of 5 . Total active range of motion (TAM) was reported in one study and averaged 139 degrees (small finger), 130 degrees (ring finger), 
105 degrees (middle finger), and 148 degrees (index). An additional study reported average percentage of TAM as small finger: $100 \%$, ring finger: $88.3 \%$, long finger $75 \%$ and, index finger $83 \%$. One study reported functional recovery as "excellent" and "good" each in one patient, and "poor" in five patients. Restoration of some protective sensation was reported in five articles, with one of the articles also reporting an average two-point discrimination of $15.4 \mathrm{~mm}$ in five patients but only pressure sensibility in three other patients.

Although there were two flap complications reported in this cohort, there was $100 \%$ flap survival. Complications included arterial crisis 12 hours after flap elevation requiring flap revision and venous congestion postoperative day 2 which resolved with suture removal. Scar widening was reported in one article which involved two patients.

\section{Discussion}

Reconstruction of digit and web space defects often requires free flaps to restore function and balance in the hand, though multilobe free flaps may offer similar outcomes to surgical syndactylization, with the same or slightly improved outcomes. Among the surgical syndactylization cohort, arterialized venous (24\%), ALT (17\%), latissimus dorsi (15\%), and medialis pedis (13\%) were the most common flaps utilized. Arterialized venous free flaps can be a good option as they are thin, create minimal donor site morbidity, and do not sacrifice arterial pedicles, as they rely purely on the subcutaneous venous network. ${ }^{9,22,25}$ However, the mechanism of flap nourishment remains unknown and they are not without complications. ${ }^{9}$ In this review, arterialized venous and venous free flaps compromised only $8 \%$ of the flaps used in this study but were responsible for $50 \%$ of the complications. These flaps were only used in the surgical syndactylization cohort with desyndactylization occurring on average 5.2 weeks (range $=3-12$ weeks) after the index operation.

While there was no major difference between timing of desyndactylization between the subgroup with complications compared with the subgroup without complications, it was 10.6 and 10.5 months, respectively; when looking exclusively at the time of desyndacytlization for arterialized venous and venous free flaps, it was 5.2 weeks. This may suggest that premature desyndactylization of arterialized venous and venous free flaps may be a factor contributing to increased complication rates. To the author's knowledge, there have been no published studies evaluating the timing of free flap desyndactylization for hand and digit reconstruction. Godina has previously demonstrated that free flap survival was the highest within 72 hours of wound coverage. ${ }^{46}$ His work has frequently been extrapolated to the upper extremity although it was based on the lower extremity. However, recent studies have contradicted these findings, demonstrating the delayed free flap reconstruction can be performed with success rates similar to those seen in early reconstruction. ${ }^{4-50}$

Lin et al described a case series of 15 patients that used shunt-restricted arterialized venous flaps for hand and digit reconstruction, one of which involved surgical syndactylization. ${ }^{25}$ The authors acknowledged that arterialized venous flaps have been known as a somewhat unreliable flap, known for ischemia and venous congestion. However, they claimed that the cause of these problems was due to unrestricted arteriovenous shunting, depriving the periphery of blood and preventing drainage. ${ }^{25}$ In their technique, they avoided retrograde flow to achieve shunt restriction by transferring the flap with antegrade flow by means of the "simulated valve" technique. This technique involved ligation of the central vein to stimulate a valve and blood still flowed to the most peripheral parts of the venous flap. Using this technique, Lin et al stated that one can reliably determine and separate the afferent and efferent pathways to ensure that no vessel simultaneously fulfills role of artery and vein simultaneously. In their series, the incidence of congestion and full-thickness necrosis was reduced. ${ }^{25}$

Venous free flap donor sites most frequently involved the volar forearm (82\%) as opposed to the dorsal foot (18\%). Takeuchi et al described a technique to harvest innervated arterialized venous flaps from the dorsal foot. Therein, the authors harvested the dorsal subcutaneous tissue off the dorsal foot with its accompanying dorsal cutaneous veins. ${ }^{30}$ The dorsal cutaneous branches of the superficial peroneal nerves were also included and coapted to the radial digital nerves after vessel anastomoses. The distal vein of the flap was anastomosed with the radial digital artery of the middle finger, and the proximal vein of the flap was anastomosed to the dorsal subcutaneous veins of the middle and ring fingers. The flap was wrapped around the degloved middle and index fingers, syndactylizing the digits. A full-thickness skin graft was used to close the donor defect. The authors reported the patient achieving good sensation and full range of motion in the fingers with no donor site complications. ${ }^{30}$

While arterialized venous free flaps may be attractive for multidigit reconstruction due to their ease of harvest with adequate pliability and thinness for digital reconstruction, their risk of complications must be considered. In this review, the surgical syndactylization cohort was the only cohort that utilized arterialized venous free flaps and it also had the highest flap complication rate of $13 \%$. This contrasts with the desyndactylization with "multilobed free flap cohort" (complication rate: $10 \%$ ), and the cohort using both techniques (complication rate: $2 \%$ ) neither of which utilized arterialized venous free flaps.

Arterial-based free flaps including the ALT and latissimus dorsi were also commonly used in the "surgical syndactylization cohort," but with less reported complications. Kim et al described a case series of seven patients with large, circumferential, and multiple-digit defects utilizing a thin latissimus dorsi perforator flap for surgical syndactylization. ${ }^{23,24}$ The authors stated that ALT and latissimus dorsi perforator flaps should be the primary flaps of choice given the ability to safely harvest large flaps, even if only one perforator is available, and vascular pedicles up to $10 \mathrm{~cm}$ can be obtained and the ability to harvest a "super-thin" flap. ${ }^{23,24}$ By not including the deep adipose layer during flap harvest, they reported that the flap can be thinned to $<5 \mathrm{~mm}$ if enough fibrofatty tissue supports the perforators, which is ideal for digit reconstruction to avoid secondary debulking procedure. Furthermore, this flap is ideally suited for distal tip or circumferential tissue loss as the super-thin flap possesses enhanced pliability to be easily 
folded. ${ }^{23,24}$ These claims are supported in their outcomes with a favorable 1-year reported DASH score average of 18.19 and $100 \%$ flap survival. ${ }^{24}$ In this review, surgical syndactylizations utilizing ALTs or latissimus dorsi flaps had accounted for $32.6 \%$ of free flaps used in this cohort with only two reported complications, including scar contracture with partial free bone resorption and sloughed tissue with exposed hardware. However, both complications where related to severe avulsion hand defects that involved three or more digits were reconstructed with more than one free flap.

In the surgical syndactylization cohort, the medialis pedis free flap was used for both multidigit volar defects, as well as a wraparound flap for neighboring digital pulp defects. The medialis pedis free flap was first described by Masquelet and Romana in 1990 as a pedicled island flap for the coverage of a local foot defect. ${ }^{51}$ Since then its versatility has been extended for multidigit reconstruction due to its matching thickness, texture, color, and sensation. ${ }^{31}$ While it can be used to revascularize devascularized finger(s) with segmental loss of the neurovascular bundle, it is not a sensory flap, typically requires skin graft closure at the donor site if the flap width is greater than $3 \mathrm{~cm}$ and is not suitable for large defects (generally $>3 \times 9 \mathrm{~cm}){ }^{12,31}$ Despite not being a sensory flap, $100 \%$ of MDFs in this cohort demonstrated normal pinprick, warm, and cold sensations, with an average static two-point discrimination of $16 \mathrm{~mm}$ and average moving two-point discrimination of $9 \mathrm{~mm}$.

This cohort demonstrated $100 \%$ free flap survival rate. However, several studies have highlighted the consequences of surgically syndactylization of digits during hand reconstruction. Tang et al argued that one-stage reconstruction is superior to two-stage reconstruction due to minimization of scarring and reducing the recovery period, potentially leading to earlier mobilization. ${ }^{39}$ Two-stage reconstruction also increases the risk of scarring due to the need for additional dissections possibly contributing to tendon adhesions and joint contractures, worsening functional outcomes. ${ }^{39}$ Lin et al further elaborated that it is difficult to desyndactylize syndactylized digits and perform debulking of the flap to reach normal skin thickness. ${ }^{26}$ Even if a very thin flap is used, Lin et al stated that very thick tissue remains under the skin and this can result in movable skin on the palmar aspect of the hand, causing inadequate power of grasp and inconvenience. ${ }^{26}$

In comparison, the use of multilobed flaps has not been well described in the literature of upper limb reconstruction. They are highly suited for upper limb reconstruction as they can be harvested to be thin and pliable, with the ability to cover multiple components on the same pedicle to facilitate three-dimensional inset of flaps. ${ }^{52}$ Branch-based multilobed flaps based on several vascular systems described in the literature include the dorsalis pedis, the deep circumflex iliac, the lateral circumflex femoral, the subscapular vessel, and the anterior tibial vessels. ${ }^{8,53-60}$

Within the multilobed free flap cohort, Tang et al accounted for $86 \%$ of the flaps described $(n=59) .{ }^{39}$ A series of 39 extensive hand and multidigit injuries were reconstructed with one-staged, combined multilobed flaps using modified designs including use of the ALT, dorsalis pedis artery (DPA), and chimeric-linking flaps. Five different microsurgical combinations of chimeric flaps systems were utilized including (1) innervated multilobed ALT flaps, (2) multilobed DPA flaps, (3) innervated multilobed ALT flap with multilobed DPA flap, (4) innervated ALT flap combined with multilobed sensate ALT flap, and (5) double-paddle bilobed ALT flap with multilobed DPA flap. All DPA donor sites were reconstructed with free ALT flaps and anterior tibial artery propeller flaps. ${ }^{39}$ The authors suggested that the use of multiple small flaps linked in a "chain-linked" flap microanastamosed chimeric system is recommended in distal hand and digital defect reconstructions. ${ }^{39}$ While no formal secondary procedures were needed for debulking or functional scar revision, four cases did require an additional procedure for web space reconstruction. ${ }^{39}$

Chen et al reported a 36-case series of microvascular free flaps based on musculocutaneous perforators for reconstruction of the hand and forearm. They reported that the thin flaps facilitated reconstructive procedures, averaging 2.3 secondary procedures. The ALTs compromised $75 \%$ of all flaps used. ${ }^{52}$ ALTs are particularly desirable as they average 2.3 perforators. $^{61-63}$ The ALTs were also most used (40\%) in the multilobed free flap cohort. Only $7 \%(n=5)$ of patients required a secondary procedure, most frequently for webspace reconstruction. In contrast, it has been reported that use of free flaps in hand reconstruction-specifically perforator flaps-have allowed for the creation of multiple skin paddles that utilize geometrical shapes to fill defects most accurately without ending up with excess wasted tissue and thus no secondary operations. In 2003, Tsai became one of the first groups to report use of a free split cutaneous flap for the reconstruction of hand defects. ${ }^{37}$ In their report, four patients with burn contractures underwent multilobed hand and dorsal web space reconstruction with free split ALT cutaneous perforator flaps. ${ }^{37}$ Means of MPJ flexion increased by 32.25 degrees, wrist palmar flexion increased by 16.5 degrees, wrist dorsiflexion increased by 9 degrees, and thumb abduction increased by 35.5 degrees and no reported complications. ${ }^{37}$

The ALT flap is not the only multilobed flap based off the circumflex femoral system. Hung et al reported a case series that included use of three free groin chimeric flaps to reconstruct two digits simultaneously and two free groin osteocutaneous flaps for thumb lengthening. These free flaps consisted of large skin paddles based off the main trunk of the superficial circumflex femoral artery. Both donor sites were closed primarily, flap survival rate was $100 \%$ and there was good finger extension and flexion at follow-up for both patients. No secondary divisions were needed and this reconstruction technique resulted in shorter recovery time and facilitated rehabilitation. ${ }^{38}$ Hung et al stated that the free groin flap not only offers a pattern for multiple finger coverage, but also an osteocutaneous pattern for thumb lengthening. ${ }^{38}$ Additionally, the free second dorsal metacarpal artery flap can be used to provide tenocutanous pattern for tendon reconstruction and soft tissue coverage simultaneously. ${ }^{38}$

\section{Surgical Syndactylization and Multilobed Free Flap Cohort} Gao et al described the feasibility and advantage of using combined island flaps based on branches of the circumflex 
scapular system for the reconstruction of multiple soft tissue defects of the hand. In a series of seven patients, they reported their experience of using the scapular lobulated combined flap based on the transverse branch, ascending branch and descending branch of the circumflex scapular vascular pedicle. ${ }^{41}$ For example, one patient experienced complete loss of soft tissue of the palm and volar side of all digits after a machine injury. Using the trilobular scapular combined flap, the ascending branch skin paddle covered the thumb, the index and long fingers were syndactylized with the transverse branch skin paddle, and the ring and small fingers were syndactylized with the descending branch skin paddle. The authors did report that the functional recovery of the affected digits in five of the seven patients was "poor," but this was likely related to the large extent of the injury involving at least four digits. With good appearance, 100\% flap survival rate and low donor site complications, the authors argue that this flap choice is probably the optimal surgical intervention for these types of hand injuries. ${ }^{41}$

Pan et al also reported hand reconstruction techniques utilizing multilobed free flaps for surgical syndactylization. In a series of eight cases, the authors described resurfacing hand defects with free iliac flaps. ${ }^{43}$ Based on the superficial circumflex iliac artery, the flap was transferred as a single unit to cover the defect and then the lateral edge of the flap was split into multiple "daughter flaps" with a length-to-width ratio of $1.5: 1{ }^{43}$ Radical debulking was required in five patients 10 to 12 weeks postsurgery at the time of digit separation. The authors reported that this was safe to perform without jeopardizing the blood supply of the flaps as all flaps survived. All patients were satisfied with the results at the final follow-up. While the dermis of the inguinal area is quite thin compared with the thigh or back, it is still bulky compared with that of the digits. However, Pan et al argue that a two-staged reconstruction process is superior to single stage reconstruction. As reported by Kimura et al and del Pinal et al, techniques to reduce bulk and achieve thin perforators include microdissection and super-thin techniques with single staged reconstruction. $^{64,65}$ Pan et al argue that these techniques require meticulous vessel dissections and a longer learning curve and that flaps can initially be thinned primarily by trimming the fatty tissue in the periphery of the flap. ${ }^{43}$

\section{Limitations}

This systematic review has several limitations. The injuries reported in the included articles varied drastically. Hand injuries spanned severities from small volar defects involving two digits to complete degloving of the soft tissue envelope distal to the palmar crease. Furthermore, the reported outcomes were highly variable and did not correlate with injury severity. Reported functional outcomes where vague at times absent of objective data. Validated questionnaires that focus on the functional outcomes of the upper extremity such as the Disabilities of the Arm, Shoulder, and Hand $(\mathrm{DASH})^{66}$ and the Michigan Hand Questionnaire, ${ }^{67}$ where only reported in three articles. This made it challenging for the authors to draw conclusions among the different tech- niques, utilizing free flaps for hand reconstruction and their associated outcomes. However, the authors meticulously categorized the articles based on their reconstructive techniques to make comparisons as accurate as possible based on the data presented. Additionally, this is the first systematic review that reports the techniques and outcomes for hand reconstruction comparing the surgical syndactylization of multiple digits with free flaps to the reconstruction of multiple digit defects with multilobed free flaps.

\section{Conclusion}

Hand and digit defects often require free flaps for reconstruction. Although free flaps for reconstruction of digital defects is technically demanding, they result in better functional and cosmetic outcomes. ${ }^{38}$ Surgical syndactylization of the digits followed by secondary desyndactylization as well as immediate desyndactylization of digits with multilobed free flaps are two reconstructive techniques to treat multidigit hand injuries. Injury severity, flap selection and technique, including desyndactylization timing, clearly play an important role in outcomes. However, acute and staged desyndactylization are two different surgical techniques that each offer their own advantages and disadvantages. Based on the $100 \%$ free flap survival rate observed in both techniques, it can be assumed that both techniques are safe to be used in the reconstructive repertoire and tailored to the defect. However, with available evidence indicating complications rates less than those of staged desyndactylization, multidigit reconstruction with multilobed free flaps may be a more desirable technique for reconstruction.

\section{Conflict of Interest}

None declared.

\section{References}

1 Saint-Cyr M, Gupta A. Indications and selection of free flaps for soft tissue coverage of the upper extremity. Hand Clin 2007;23 (01):37-48

2 Miller EA, Friedrich J. Soft tissue coverage of the hand and upper extremity: the reconstructive elevator. J Hand Surg Am 2016;41 (07):782-792

3 Gupta A, Lakhiani C, Lim BH, et al. Free tissue transfer to the traumatized upper extremity: Risk factors for postoperative complications in 282 cases. J Plast Reconstr Aesthet Surg 2015; 68(09):1184-1190

4 Chen HC, Buchman MT, Wei FC. Free flaps for soft tissue coverage in the hand and fingers. Hand Clin 1999;15(04):541-554

5 Giessler GA, Erdmann D, Germann G. Soft tissue coverage in devastating hand injuries. Hand Clin 2003;19(01):63-71, vi

6 Slavin SA, Upton J, Kaplan WD, Van den Abbeele AD. An investigation of lymphatic function following free-tissue transfer. Plast Reconstr Surg 1997;99(03):730-741, discussion 742-743

7 Yu G, Lei HY, Guo S, Yu H, Huang JH. Treatment of degloving injury of three fingers with an anterolateral thigh flap. Chin J Traumatol 2011;14(02):126-128

8 Xie G, Hu Z, Miao C, Chen W, Mei L. The free triple chimeric dorsalis pedis flaps for repair of multifinger soft tissue defects: a report of two cases. Microsurgery 2013;33(08):660-666

9 Trovato MJ, Brooks D, Buntic RF, Buncke GM. Simultaneous coverage of two separate dorsal digital defects with a syndactylizing venous free flap. Microsurgery 2008;28(04):248-251 
10 Cho BC, Lee JH, Weinzweig N, Baik BS. Use of the free innervated dorsalis pedis tendocutaneous flap in composite hand reconstruction. Ann Plast Surg 1998;40(03):268-276

11 Eren F, Oksuz S, Karagöz H, Melikoğlu C, Ulkur E. Multi-digit contracture release using medial sural artery perforator flap with syndactylization-desyndactylization method. Hippokratia 2015; 19(04):366-368

12 Tsai FC, Cheng MH, Chen HC, Wei FC. Microsurgical medialis pedis flaps for reconstruction of soft-tissue defects in the hand. Ann Plast Surg 2002;48(01):41-47

13 Giessler GA, Schmidt AB, Germann G, Pelzer M. The role of fabricated chimeric free flaps in reconstruction of devastating hand and forearm injuries. J Reconstr Microsurg 2011;27(09): 567-573

14 Woo SH, Kim KC, Lee GJ, et al. A retrospective analysis of 154 arterialized venous flaps for hand reconstruction: an 11-year experience. Plast Reconstr Surg 2007;119(06):1823-1838

15 Zheng H, Liu J, Dai X, Schilling AF. Free conjoined or chimeric medial sural artery perforator flap for the reconstruction of multiple defects in hand. J Plast Reconstr Aesthet Surg 2015;68 (04):565-570

16 Agarwal JP, Agarwal S, Adler N, Gottlieb LJ. Refining the intrinsic chimera flap: a review. Ann Plast Surg 2009;63(04):462-467

17 Yan H, Zhang F, Akdemir O, et al. Clinical applications of venous flaps in the reconstruction of hands and fingers. Arch Orthop Trauma Surg 2011;131(01):65-74

18 Lai CL, Ou KW, Chiu WK, et al. Reconstruction of the complete loss of upper and lower lips with a chimeric anterolateral thigh flap: a case report. Microsurgery 2012;32(01):60-63

19 Moher D, Liberati A, Tetzlaff J, Altman DG, Group PPRISMA Group. Preferred reporting items for systematic reviews and meta-analyses: the PRISMA statement. BMJ 2009;339:b2535

20 Iñigo $F$, Gargollo $C$. Secondary coverage of the hand using a dorsalis pedis plus first web space free flap. J Reconstr Microsurg 1992;8(06):461-465

21 Inoue G, Suzuki K. Arterialized venous flap for treating multiple skin defects of the hand. Plast Reconstr Surg 1993;91(02):299-302, discussion 303-306

22 Kantarci U, Cepel S, Gürbüz C. Venous free flaps for reconstruction of skin defects of the hand. Microsurgery 1998;18(03):166-169

$23 \mathrm{Kim}$ KS, Kim ES, Kim DY, Lee SY, Cho BH. Resurfacing of a totally degloved hand using thin perforator-based cutaneous free flaps. Ann Plast Surg 2003;50(01):77-81

$24 \mathrm{Kim}$ SW, Lee HJ, Kim JT, Kim YH. Multiple-digit resurfacing using a thin latissimus dorsi perforator flap. J Plast Reconstr Aesthet Surg 2014;67(01):74-80

25 Lin YT, Henry SL, Lin CH, et al. The shunt-restricted arterialized venous flap for hand/digit reconstruction: enhanced perfusion, decreased congestion, and improved reliability. J Trauma 2010;69 (02):399-404

26 Lin TS. One-stage debulking procedure after flap reconstruction for degloving injury of the hand. J Plast Reconstr Aesthet Surg 2016;69(05):646-651

27 Ninkovíc MM, Schwabegger AH, Wechselberger G, Anderl H. Reconstruction of large palmar defects of the hand using free flaps. J Hand Surg [Br] 1997;22(05):623-630

28 Sakai S. Free flap from the flexor aspect of the wrist for resurfacing defects of the hand and fingers. Plast Reconstr Surg 2003;111(04): 1412-1420, discussion 1421-1422

29 Shilov BL. Resurfacing of the damaged hand by the prefabricated free flap including serratus fascia: a case report. Microsurgery 1995;16(06):370-372

30 Takeuchi M, Sakurai H, Sasaki K, Nozaki M. Treatment of finger avulsion injuries with innervated arterialized venous flaps. Plast Reconstr Surg 2000;106(04):881-885

31 Wong SS, Wang ML, Su MS, Wei FC. Free medialis pedis flap as a coverage and flow-through flap in hand and digit reconstruction.J Trauma 1999;47(04):738-743
32 Woo SH, Jeong JH, Seul JH. Resurfacing relatively large skin defects of the hand using arterialized venous flaps. J Hand Surg [Br] 1996; 21(02):222-229

33 Vogt PM, Mett TR, Bingoel AS, Jokuszies A, Krezdorn N. Reconstruction of basic hand function by a free osteocutaneous sparepart flap from the lower leg after septicemia: a case report. Hand (N Y) 2020;1558944720906499;

$34 \mathrm{Xu} \mathrm{L,} \mathrm{Xu} \mathrm{J,} \mathrm{Shou} \mathrm{K,} \mathrm{Rui} \mathrm{Y.} \mathrm{Repair} \mathrm{of} \mathrm{avulsion} \mathrm{injury} \mathrm{of} \mathrm{the} \mathrm{whole} \mathrm{hand}$ with single-stage transfer of five combined tissues: case report. J Reconstr Microsurg 2003;19(02):79-84, discussion 85-86

35 Chai YM, Wang CY, Wen G, Zeng BF, Cai PH, Han P. Combined medialis pedis and medial plantar fasciocutaneous flaps based on the medial plantar pedicle for reconstruction of complex soft tissue defects in the hand. Microsurgery 2011;31(01):45-50

36 Chung KC, Tong L. Use of three free flaps based on a single vascular pedicle for complex hand reconstruction in an electrical burn injury: a case report. J Hand Surg Am 2001;26(05):956-961

37 Tsai FC, Yang JY, Mardini S, Chuang SS, Wei FC. Free split-cutaneous perforator flaps procured using a three-dimensional harvest technique for the reconstruction of postburn contracture defects. Plast Reconstr Surg 2004;113(01):185-193, discussion 194-195

38 Hung MH, Huang KF, Chiu HY, Chao WN. Experience in Reconstruction for Small Digital Defects With Free Flaps. Ann Plast Surg 2016;76(Suppl 1):S48-S54

39 Tang L, Pafitanis G, Yang P, et al. Combined multi-lobed flaps: a series of 39 extensive hand and multi-digit injuries one-staged reconstructions using modified designs of ALT, DPA and chimeric linking flaps. Injury 2017;48(07):1527-1535

40 Caulfield RH, Maleki-Tabrizi A, Birch J, Ramakrishnan V. Salvage of finger length in septicemic necrosis using 3 free flaps from a single anterolateral thigh donor site. Ann Plast Surg 2008;60(06): 623-625

41 Gao W, Hong J, Li Z, Chen X. Hand reconstruction with lobulated combined flaps based on the circumflex scapular pedicle. Microsurgery 2008;28(05):355-360

42 Okada M, Saito H, Kazuki K, Nakamura H. Combined medialis pedis and medial plantar fasciocutaneous flaps for coverage of soft tissue defects of multiple adjacent fingers. Microsurgery 2014;34(06):454-458

43 Pan ZH, Jiang PP, Xue S. Free iliac flap for treating multiple skin defects of the hand and digits. J Hand Surg Eur Vol 2013;38(09): 952-958

44 Pan ZH, Jiang PP, Xue S, Li H, Wang JL. Restoration of basic hand function following devastating hand injuries using a microsurgically fabricated chimeric iliac osteocutaneous flap. J Plast Reconstr Aesthet Surg 2017;70(06):723-728

45 Ulrich D, Pallua N. Treatment of avulsion injury of three fingers with a compound thoracodorsal artery perforator flap including serratus anterior fascia. Microsurgery 2009;29(07):556-559

46 Godina M. Early microsurgical reconstruction of complex trauma of the extremities. Plast Reconstr Surg 1986;78(03):285-292

47 Francel TJ, Vander Kolk CA, Hoopes JE, Manson PN, Yaremchuk MJ. Microvascular soft-tissue transplantation for reconstruction of acute open tibial fractures: timing of coverage and long-term functional results. Plast Reconstr Surg 1992;89(03):478-487, discussion 488-489

48 Ofer N, Baumeister S, Megerle K, Germann G, Sauerbier M. Current concepts of microvascular reconstruction for limb salvage in electrical burn injuries. J Plast Reconstr Aesthet Surg 2007;60 (07):724-730

49 Karanas YL, Nigriny J, Chang J. The timing of microsurgical reconstruction in lower extremity trauma. Microsurgery 2008; 28(08):632-634

50 Kumar AR, Grewal NS, Chung TL, Bradley JP. Lessons from the modern battlefield: successful upper extremity injury reconstruction in the subacute period. J Trauma 2009;67(04):752-757

51 Masquelet AC, Romana MC. The medialis pedis flap: a new fasciocutaneous flap. Plast Reconstr Surg 1990;85(05):765-772 
52 Chen HC, Tang YB, Mardini S, Tsai BW. Reconstruction of the hand and upper limb with free flaps based on musculocutaneous perforators. Microsurgery 2004;24(04):270-280

53 Lai CS, Lin SD, Yang CC. The reverse digital artery flap for fingertip reconstruction. Ann Plast Surg 1989;22(06):495-500

54 Lai CH, Lai CS, Huang SH, Lin SD, Chang KP. Free medial plantar artery perforator flaps for the resurfacing of thumb defects. Ann Plast Surg 2010;65(06):535-540

55 Flatt AE. The thenar flap. J Bone Joint Surg Br 1957;39-B(01): 80-85

56 Hastings H II. Dual innervated index to thumb cross finger or island flap reconstruction. Microsurgery 1987;8(03):168-172

57 Shi SM, Lu YP. Island skin flap with neurovascular pedicle from the dorsum of the index finger for reconstruction of the thumb. Microsurgery 1994;15(02):145-148

58 Inoue T, Kobayashi M, Harashina T. Finger pulp reconstruction with a free sensory medial plantar flap. Br J Plast Surg 1988;41 (06):657-659

$59 \mathrm{Gu} \mathrm{JH}$, Jeong SH. Radical resection of a venous malformation in middle finger and immediate reconstruction using medial plantar artery perforator flap: a case report. Microsurgery 2012;32(02): 148-152

60 Chuang DC, Colony LH, Chen HC, Wei FC. Groin flap design and versatility. Plast Reconstr Surg 1989;84(01):100-107
61 Kimata Y, Uchiyama K, Ebihara S, et al. Versatility of the free anterolateral thigh flap for reconstruction of head and neck defects. Arch Otolaryngol Head Neck Surg 1997;123(12): 1325-1331

62 Kimata Y, Uchiyama K, Ebihara S, Nakatsuka T, Harii K. Anatomic variations and technical problems of the anterolateral thigh flap: a report of 74 cases. Plast Reconstr Surg 1998;102(05):1517-1523

63 Koshima I, Fukuda H, Yamamoto H, Moriguchi T, Soeda S, Ohta S. Free anterolateral thigh flaps for reconstruction of head and neck defects. Plast Reconstr Surg 1993;92(03):421-428, discussion 429-430

64 Kimura N, Saitoh M. Free microdissected thin groin flap design with an extended vascular pedicle. Plast Reconstr Surg 2006;117 (03):986-992

65 del Piñal F, García-Bernal FJ, Studer A, Ayala H, Cagigal L, Regalado J. Super-thinned iliac flap for major defects on the elbow and wrist flexion creases. J Hand Surg Am 2008;33(10):1899-1904

66 Hudak PL, Amadio PC, Bombardier CThe Upper Extremity Collaborative Group (UECG). Development of an upper extremity outcome measure: the DASH (disabilities of the arm, shoulder and hand) [corrected]. Am J Ind Med 1996;29(06):602-608

67 Chung KC, Pillsbury MS, Walters MR, Hayward RA. Reliability and validity testing of the Michigan Hand Outcomes Questionnaire. J Hand Surg Am 1998;23(04):575-587 